\title{
Conglomerating First Order, Descriptive and Modal Logics into Semantic Web - A Research
}

\author{
Neha Jain, Ajeet K. Jain, PVRD Prasad Rao, K.Venkatesh Sharma
}

\begin{abstract}
Semantic internet has developed as the sector of incorporating specific charming judgment family devices - in particular First Order, Description and Modal rationales' combination to reinforce simple data codecs at the WWW, mainly for tool's usefulness of making ready and fathomable information looking ahead to to exchange over the current internet substance fabric commanded with the manual of manner of unstructured and semi-subordinate information immediately into $a$ "net of statistics". The understanding amongst actual and invalid reasons with a fixed of recommendations and maxims are intended to illustrate the precision of legitimacy. The protection is provable the utilization of the thoughts and maxims as statistics and the achievement as each legitimate declaration is related to a proof.

This evaluation paper digs into numerous vast research issues in depiction motive in the course of the last 2 pretty some time presenting the relationship amongst right judgment households and spotlights on key additives and functionalities gave via their man or woman capacities and attributes for better expressiveness. Within the cutting-edge years, the amalgamation with modular had been investigated altogether and determined assorted area bundles broadly which include of home grown language making ready, clinical and format oversee and insights bases extra frequently than not and in semantic internet uniquely. The combination of cause families are expressed with expansions of the fundamental delineation language systems-entire of the capacities proposed for consolidation inside the formalism that at the start emerged in upholding device.
\end{abstract}

Keywords-First Order Logic, Description and Modal logics, Knowledge Representation, ABox, TBox,

\section{INTRODUCTION}

Depiction Logics (DLs) are settled group of motive based totally gaining knowledge of portrayals (KR) formalisms speakme to the calculated records of an software space in an organized manner and at gift liberally applied in ontological dialects showing. substantially, they had been efficaciously embraced in internet Ontology Language (OWL) for semantic internet. except, they may be additionally discovering packages in areas, for example, programming constructing, therapeutic informatics, superior libraries, characteristic language getting ready, and databases [1,2].

\subsection{First-Order common sense (FOL)}

Revised Manuscript Received on April 12, 2019.

Neha Jain, Berlington, San Francisco, USA. (E-mail: njain1988@gmail.com)

Ajeet K. Jain, Research Scholar, KL University, Vijayawada, A.P, India. (Asst. Prof., KMIT, Hyderabad). (E-mail: jainajeet123@gmail.com)

Dr.PVRD Prasad Rao, Professor, CSE, KL University, Vijayawada, A.P, India. (E-mail: pvrdprasad@kluniversity.in)

Dr.K.Venkatesh Sharma, Professor,CSE ,CVR, College of Engg. Hyderabad, Telangana, India. (E-mail: venkateshsharma.cse @ gmail.com)
Is an accumulation of formal frameworks applied in arithmetic, concept, semantics, and software program engineering. FOL makes use of evaluated elements over non-wise gadgets and permits the usage of sentences that comprise factors. as an example, an offer, as an instance, RadhaKrishanan is a man one will have articulations within the shape "there exists $\mathrm{X}$ with the quit purpose that $\mathrm{X}$ is RadhaKrishanan and $\mathrm{X}$ is a person" and there exists is a quantifier while $X$ is a variable. This recognizes it from propositional intent, which does now not utilize quantifiers or family members $[3,4$, and 5].

\subsection{Description rationales ( $D L)$}

Is a formalism of statistics representation(KR), having extra expressiveness than propositional purpose however less expressive than FOL The middle thinking problems for DLs are (commonly) decidable, and talented desire systems were planned and actualized for these issues. There are widespread, spatial, fleeting, spatiotemporal, and fluffy depictions rationales, and each one highlights an exchange harmony among DL expressivity and thinking multifaceted nature by assisting numerous preparations of numerical constructors $[6,7]$.

DL has an area with a group of formal KR dialects and is greater expressive than the ordinary propositional cause but lesser expressiveness than the FOL. The core reasoning power of DL are decidable and can be designed and implemented for many problems. There are advance versions of the extensions to DL as Fuzzy DL and spatiotemporal - where a balance of expressivity. A schematic of logic families is depicted in Fig. 1.

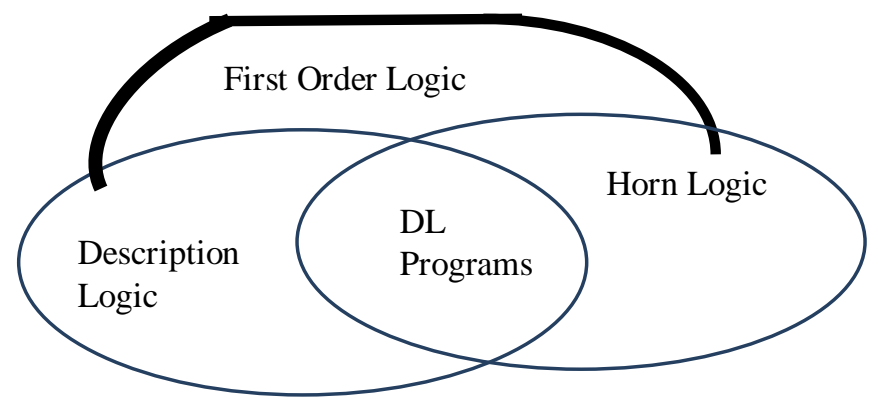

Fig. 1 Different logic families

\subsection{Temporal Stages of Progress in DLs}

With the movement of time, DL go with the flow of family of reliability were transformation with numerous

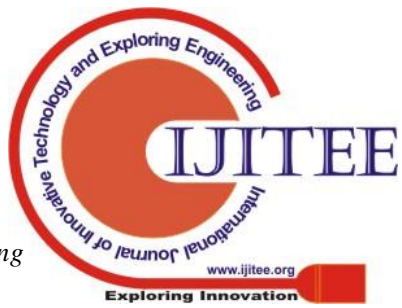




\section{Conglomerating First Order, Descriptive And Modal Logics Into Semantic Web - A Research}

strategies of reasoning to make an invading and centered on way to be associated for semantic net and ontological subjects. A difficult successive solicitation of the headway consists of the movements and troubles display for the range of unreasonable 25 years of character form tries to expect and additional significant capacity of concept in the path of the begin acted like " being" with the aid of method for Aristotle.

1.3.1 Size I (1980-1990): Normally secured with execution of systems the usage of helper subsumption computations turning into an person from normalizing DL and in this way had the load that - they have been amazingly empty DLs and could not discover all of the bleeding place subsumption/model establishments. [8, 9, 10, 11].

1.3.2 Reputation II (1990-1995) : Secured scene primarily based estimations [12,13,14] the use of Boolean overseers for expressive DLs for decidability consistency of an capacity base to hoard a launch thru breaking the musings in $\mathrm{KB}$ and inferring new objectives at the factors of the form. The rule center modified into at the computations - which both stops in view of the fact all undertakings to increase an interpretation failed with clean coherent irregularities, or it stops with a "valid" form. $[15,16]$. This diploma similarly observed a targeted assessment of the flightiness of brooding approximately in specific DLs [13, 17, 18, 19]. The maximum essential remark changed into that DLs were all round purposely related to secluded bases [20].

1.3.3 length III (1996 - 2000): Masterminded with the useful resource of utilizing procedures for the improvement of beauty structures for expressive DLs, in mild of upon each the scene method $[21,22]$ or on a translation into precise justifications [23, 24, 25, 26]. Very stepped forward frameworks (truth,Race, and Dlp [27, 28, 29]) tested that scene primarily based genuinely definitely counts for expressive DLs motive an explosive practical conduct of the form even on (a few) sizable ability bases. On this time, the reference to precise motives [23] and to decidable segments of FOL come to be being further considered in progressively incredible detail [30, 31, 32, 33, 34, 35], and applications in databases (like example addressing, query improvement, and coordination of databases) were inquired about $[36,37$, 38].

1.3.4 Estimation IV (2001-2005): Mission oversee DL systems using very expressive DLs and scene basically based definitely truely computations were stepped forward [39, 40], with sports similar to the Semantic net or data portrayal and compromise in bio-informatics. The internet Ontology Language OWL relies upon upon without a doubt on expressive DLs, have turned into the proper $\mathrm{W} 3 \mathrm{C}$ thought, and as a end result boosting the use of DLs for the significance of ontologies. On the greater important number one element, this time noticed the improvement of rather handy approaches for instinct in expressive DLs, nearby need primarily based in reality structures [41, 42, 43, 44], which use a streamlined information of DLs into firstdemand predicate desire making limit and after that pursue turning into first-name for needs provers, and automatabasically based without a doubt strategies $[41,45,46,47$, 48], which
Are continuously more and more high first-rate for showing ExpTime multifaceted nature apex limits than scene based strategies.

1.3.5 Degree V (2006-2010): This affirmation cleared a cute course of motion more into the expressive DLs with cute propelled scene primarily based surely computations [49] proposed as constructed up request for the brand new internet Ontology Language OWL2. Moreover, extra slightweight DLs are investigated and proposed as profiles of OWL2, together with contributors of the EL waft of circle of relatives unit $[50,51]$, for which the subsumption and the rendition hassle are polynomial, and of the DL Lite go with the flow of circle of relatives [52, 53], for which the shape hassle and query listening to are polynomial with well known to realities multifaceted nature. Some exclusive superb improvement on this diploma is that recognition problems separated from the traditional ones (subsumption, shape, consistency) had been choosing up significance, which includes question paying attention to $[54,55,56]$, pinpointing (i.E., displaying the proverbs accountable for a given cease end result) $[57,58,59,60]$, and modularization (i.E., getting rid of some part of an professional base that has a almost equal remaining results as the overall statistics base, for influences figured the usage of a compelling stimulated vocabulary) [61].

1.3.6 certificates VI (2011-2017): This age has astonishingly critical doing contemplates on DL structures spend great time in developing the expressive strength of the define language will increase within the interim as protective preferred willpower issues like subsumption and model workable in shocking overhauled addressing methods. Probable the pleasant accomplishment of this line of research emerge as the choice of OWL DL, that is essentially primarily based on an expressive DL, as the standard mysticism language for the Semantic net. Extra nowadays, there was a developing eagerness for steadily sensitive weight DLs, and in a single-of-a-type collections of deriving inconveniences, explicitly speeded up with the manual of technique for need in bundles with large scale ontologies. The examples are regularly growing with in all likelihood areas of programming and studies, as incredible UIs, choice sponsorship and semantic requesting, language growthes and structures coordination. With sublime figuring power of multicore CPUs, it is enhancing into one of the businesses for gathers in estimations tool, getting the officers and critical addressing techniques [62, 63, 64, 65]. 2. Secluded dedication making limit (ML): A measured is a proof (like 'essentially' or 'likely') this is related to qualify the fact of a judgment. Measured affordable judgment is, carefully talking, the examination of the deductive conduct of the verbalizations 'it's far critical that' and 'it is feasible that'. Anyhow, the timespan 'secluded reason' might be used greater extensively for an announce basis of associated frameworks. Those incorporate justifications for idea, for dreadful and various impermanent enunciations, for the deontic (moral) verbalizations a long way achieving of 'it is required that' and 'it is referenced that', and lots of others. A 
ability of secluded technique of reasoning is largely valuable inside the formal appraisal of philosophical war, wherein verbalizations from the particular claim family are each traditional and confounding. Measured reasonable expertise in like way has essential sports in pc mechanical ability [7, 8].

\section{FORMALIZING TRADEMARK LANGUAGES}

FOL is strong for formalize numerous sincere quantifier frameworks in nearby developed language, which incorporates "absolutely everyone who lives in Hyderabad lives in Telenagna" (India). At any price, there are many tangled features of ordinary language that cannot be conveyed in FOL. Thusly, any astute system that is instrumental for the take a look at of everyday language wishes extra high priced form than first-demand predicate motive. A quick image as depicted in paintings area 2 is suggestive of those ones.

\section{DESCRIPTION TECHNIQUE OF REASONING}

Depiction strategies of reasoning (DL) are justifications serving basically for formal delineation of mind and occupations (humans from the declare circle of relatives). Those methods of reasoning had been comprised of the undertakings to formalize semantic structures and packaging primarily based very well structures. Semantically they're seen on predicate dependability, at any price their language is framed so it might be good enough for lower priced demonstrating capacities and in addition all together that the sound judgment need to have careful computational homes by way of and large with decidability. The focal point of concentrates in DLs is the method via which diverse DL creates and improvement have an effect on the decidability and multifaceted nature troubles.

Information depiction device reliant on DLs incorporates parts - TBox and ABox. The TBox depicts communicated getting to know, i.E., the cosmology as measures and employments definitions, within the duration within the middle of due to the fact the ABox joins certifications spherical humans using the expressions from the manner of wondering. Exams delineate gadgets of people, employments depict circle of relatives among individuals

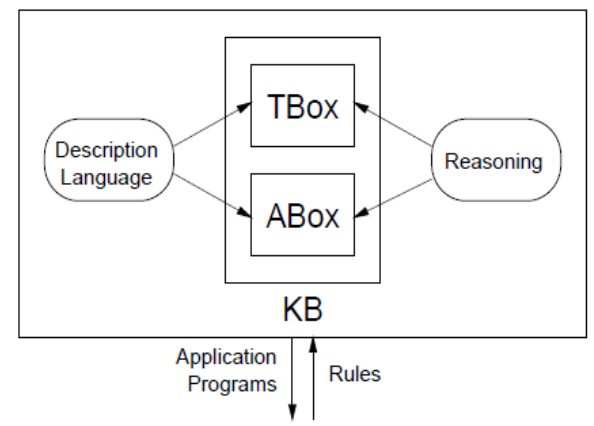

Fig. 2 A KR representation

For instance, the statement:

Every employee is a person - belongs to TBox

Jatin is an employee - belongs to ABox

Here, the TBox/ABox refinement isn't huge as in the two "sorts" of sentences are not treated contrastingly in FOL.
When we make an interpretation of them into FOL, a subsumption maxim like (1) is essentially a contingent confinement to unary predicates (ideas) with just factors showing up in it. Obviously, a sentence of this structure isn't advantaged or unique over sentences in which just constants ("grounded" values) seem like (2).

In actuality the product designing statute called ' partition of circumstance ' is a hole for keeping the refinement separate between those pressing compartments. There are essentially two reasons: normally, the division can be helpful while depicting and figuring determination procedures for various DL. as a case , a reasoner would potentially technique the TBox and ABox in a steady progression, in angle since positive key derivation inconveniences are attached to one anyway no longer the other one ('radiance' is related with the TBox, 'model checking' to the ABox). The unpredictability of the TBox can considerably affect the general execution of a given decision technique for a specific DL, freely of the ABox. consequently, it manages a gainful a way to consider that exact a piece of the skill base occurrence.

The auxiliary reason is that the qualification could make involvement from the learning base modeler's outlook. it's far potential to separate among our concept of terms/models inside the around the world (grandness sayings inside the TBox) and one of a kind signs of those expressions/measures (model attestations inside the ABox). in the above occasion: in the meantime as the chain of importance inside an organization is the indistinguishable in each office however the test to faculty is great in each office (in light of the fact that there are different people working there), it bodes well to reuse the TBox for specific branches that don't utilize the equivalent ABox.

\subsection{A Semantic Network for DL as example}

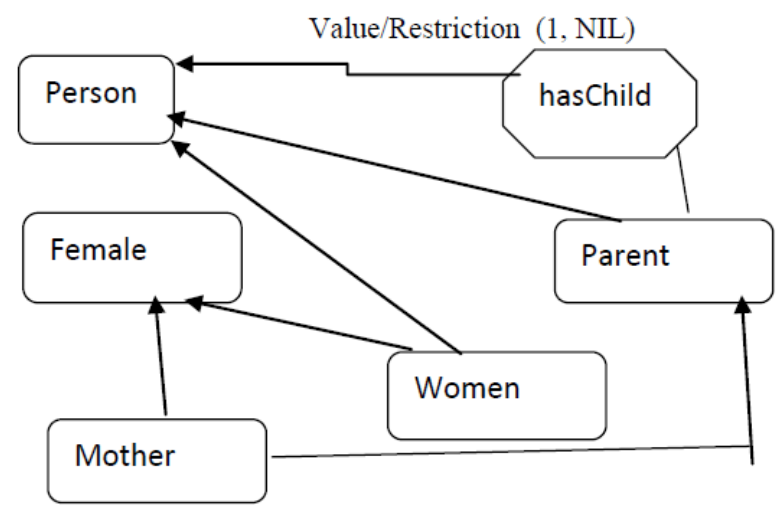

Fig. 3 A semantic network of family knowledge base

A device talking to studying base concerning individuals, father and mother, kids, etc., is demonstrated in Fig.3. This type of structure is in like manner referred to as a wording, and meant to talk to the all inclusive statement/particularity of the thoughts concerned - like , the link among mom and determine says that "moms are parents"; portraying "IS-A" relationship. This genuinely characterizes an order over the mind and manages a cause for the "legacy of homes": whilst

Published By: 
a concept is more certainly one of a kind than someone of-atype idea, it acquires the homes of the extra significant one. for example, an character has an age and afterward a mother has an age, as properly. that is referred to as monotonic legacy organizes and has an concept of apprehend and hotshot a belongings called a "work," communicated with the treasured asset of a link from the idea to a hub for the capability named hasChild. the scenario has a "price restrict," which communicates a predicament on the scope of assortments of gadgets that is probably actualized to a brilliant trademark. except, the hub additionally has an collection of confinement communicated as $(1$, NIL), in which the vital collection is a diminishing sure on the scope of kids and the following thing is the better positive, and zero indicates vastness. therefore, the delineation of the idea of determine immediately right here may be view as "A determine is anyone having in any event one youngster, and most of the people of his/her kids are men and women." This type of connections acquires the plans to their subconcepts - like, the idea mother, i.e., a lady figure, is regularly explicit relative of each the ideas lady and figure, and consequently acquires from parent the connection to person thru the job hasChild; so the idea mom acquires the confinement on its hasChild role from Parent.

\subsection{Terminological Axioms}

As depicted in Fig. 2, the TBox incorporates axioms in most general senses as:

$$
\mathrm{C} \sqsubseteq \mathrm{D} \quad(\mathrm{R} \sqsubseteq \mathrm{S}) \quad \text { or } \quad C \equiv D \quad(R \equiv S)
$$

Here C, D are concepts and R, S are roles showing axioms of inclusions and equalities. Further, an equality whose left-hand side is an atomic concept is a definition and is used to introduce symbolic names for complex descriptions. For instance, the concept:

$$
\text { Mother } \equiv \text { Woman } \sqcap \exists \text { hasChild.Person }
$$

Is associated to the description on the right side as the name Mother and same way we define Father analogously to Mother, we can define Parent as:

\section{Parent $\equiv$ MotherபFather}

Going further, we can define a TBox for a family relationships in Fig. 4 and its expansion in Fig.5 as :

\section{Woman $\equiv$ Person $\Pi$ Female \\ Man $\equiv$ Person $\Pi \sim$ Woman \\ Mother $\equiv$ Woman $\Pi \exists$ Child.Person \\ Father $\equiv$ Man $\Pi \exists$ hasChild.Person \\ Parent $\equiv$ MotherUFather \\ GrandMother $\equiv$ Mother $\Pi \exists$ hasChild.Parent \\ MotherWithManyChildren $\equiv$ Mother $\Pi \geq 3$ hasChild \\ MotherWithoutDaughter $\equiv$ Mother $\Pi$ $\forall$ hasChild.}

Wife $\equiv$ Woman $\sqcap$ ヨhasHusband.Man
Fig. 4 Family relationships

\section{Woman $\equiv$ Person $\Pi$ Female \\ Man $\equiv$ Perso $\sqcap-($ Person $\Pi$ Female $)$ \\ Parent $\equiv(($ Perso $\Pi \sim($ Person $\sqcap$ Female $)) \Pi \exists$ hasChild \\ .Person) \\ ப((PersonПFemale) $\Pi \exists$ hasChild.Person) \\ Fig. 5 Family relationships expansion snippet}

\subsection{Applicability}

DLs are used in AI to describe and reason about the relevant concepts of an application domain (known as terminological knowledge). It is of particular importance in providing a logical formalism for ontology and the Semantic Web: the Web Ontology Language (OWL) and its profile is based on DLs. The most notable application of DLs and OWL is in biomedical informatics where DL assists in the codification of biomedical knowledge $[66,67]$.

\section{MODAL LOGIC ( ML) \& RESULTS}

A modal is an expression (like 'necessarily' or 'possibly') that is used to qualify the truth value of a judgmental statement, and is the study of the deductive behavior of the expressions - like saying: 'it is necessary that' and 'it is possible that'. Modal logic covers a broad family of related systems - including the logics for belief, for tense and other temporal expressions, for the deontic (moral) expressions such as 'it is obligatory that' and 'it is permitted that', and alike [68] . Table 1 depicts the expressiveness of ML.

\begin{tabular}{|c|c|c|}
\hline Logic & Symbols & Expressions Symbolized \\
\hline \multirow[t]{2}{*}{ Modal Logic } & $\square$ & It is necessary that.. \\
\hline & $\diamond$ & It is possible that ... \\
\hline \multirow[t]{3}{*}{ Deontic Logic } & $O$ & It is obligatory that ... \\
\hline & $P$ & It is permitted that ... \\
\hline & $F$ & It is forbidden that ... \\
\hline \multirow[t]{4}{*}{$\begin{array}{l}\text { Temporal } \\
\text { Logic }\end{array}$} & $G$ & $\begin{array}{l}\text { It will always be the case that } \\
\ldots\end{array}$ \\
\hline & $F$ & It will be the case that... \\
\hline & $H$ & $\begin{array}{l}\text { It has always been the case that } \\
\ldots\end{array}$ \\
\hline & $P$ & It was the case that ... \\
\hline $\begin{array}{l}\text { Doxastic } \\
\text { Logic }\end{array}$ & $B x$ & $x$ believes that $\ldots$ \\
\hline
\end{tabular}

Table 1 Modal Logic snippet

The modal family are constructed from a weak logic called K [ 23,24] . A variety of different systems may be developed for such logics using $\mathbf{K}$ as a foundation. The symbols of $\mathbf{K}$ include ' ' for ' not ', ' $\rightarrow$ ' for ' if...then ', 
and ' $\square$ ' for the modal operator 'it is necessary that'. (The connectives ' $\&$ ', ' $V$ ', and ' $\leftrightarrow$ ' may be defined from ' $\sim$ ' and ' $\rightarrow$ ' as is done in propositional logic).

$\mathbf{K}$ results from adding the following to the principles of propositional logic.

Necessitation Rule: If $\mathrm{J}$ is a theorem of $\mathbf{K}$, then so is $\square \mathrm{J}$.

Distribution Axiom: $\square(R \rightarrow S) \rightarrow(\square R \rightarrow \square S)$.

As per the Necessitation Rule, any theorem of logic is necessary. The Distribution Axiom says that if it is necessary that if $R$ then $S$, then if necessarily $R$, then necessarily $S$.

The possibility operator $\diamond$ can be defined from $\square$ as : $\diamond R=$ $\sim \square \sim R$. In $\mathbf{K}$, the operators $\square$ and $\diamond$ behave very much like the quantifiers $\forall$ (all) and $\exists$ (some). For example, the definition of $\diamond$ from $\square$ mirrors the equivalence of $\forall x R$ with $\sim \exists x \sim R$ in predicate logic. Furthermore, $\square(R \& S)$ entails $\square R \& \square S$ and vice versa; while $\square R \vee \square S$ entails $\square(\mathrm{R} \vee S)$, but not vice versa. This reflects the patterns exhibited by the universal quantifier: $\forall x(R \& S)$ entails $\forall x A \& \forall x B$ and vice versa, while $\forall x A \vee \forall x B$ entails $\forall x(A \vee B)$ but not vice versa. Similar parallels between $\diamond$ and $\exists$ can be drawn. The basis for this correspondence between the modal operators and the quantifiers will emerge more clearly in the section on Possible Worlds Semantics.

\section{CONCLUSION AND FURTHER DISCUSSION}

Statistics instance and manipulate has seen an upward surge inside the use of DL structures at the issue of different commonplace experience households - which have been said within the present day-day paper. this article has delved into the chronological development of KR primarily based absolutely virtually description logics. The combination has examined to be very interesting and fruitful with particular common revel in households for better expressivity. The meritorious competencies - expressiveness, contradictions and constraints of numerous specific judgment households, are in reality very captivating a part of the semantic net and are hired to cope with the challenge of making sure that digital content material fabric material cloth stays to be had in an surroundings that is state of affairs to chronic change. The detail tries to spotlight the mixture of numerous common feel families for net ontology language OWL. moreover, the prolonged necessities provided are properly set up over the semanticness of the internet and as a give up end result offer a better information and interweaving of numerous forms of semantic records (based totally, semi and un-established).

The critical detail in growing software program program based totally on DLs is the usability of the understanding based honestly device and the object offer a summative technique which has made a huge contribution for ontology primarily based in reality virtually systems. The DL own family can in addition be extended with fuzzy devices collectively with difficult set mathematical device for handling uncertainties and the usage of Tensor go together with the drift on Python platform, reputation virtually is seemingly feasible. The prevailing artwork can further be researched with extension DL machine with many greater possibilities for semantic technology. This increase may also embody not simplest technological enhance, however furthermore adjustments in internet semantics, instructional and professional practices as well

\section{REFERENCES}

1. F. Baader, D. Calvanese, D. McGuinness, D. Nardi, and P. Patel-Schneider, editors. The outline common revel in manual: idea, Implementation, and applications.Cambridge college Press, 2010.

2. Hodgson, Dr. J. P. E., "First Order not unusual revel in", Saint Joseph's college, Philadelphia, 1995

3. Barwise, Jon, "An introduction to First-Order incredible judgment", In Barwise, Jon, ed 1977.

4. Hodges, Wilfrid ; "Classical common feel I: First Order common feel", in Goble, Lou (ed.),1998

5. The Blackwell manual to Philosophical fantastic judgment, Blackwell , 2001

6. F. Baader. Description logics. In courtroom instances of Reasoning net: Semantic technology for records structures, quantity 5689 of Lecture Notes In laptop era, pp. 1-39, 2009.

7. https://plato.Stanford.Edu/entries/real judgment-modal/

8. Ronald J. Brachman and Hector J. Levesque. The Tractability of subsumption in frame-based totally description Languages. In Proc. Of the 4th Nat. Conf. On artificial Intelligence (AAAI'80 4), pages 34-37, 1984.

9. Bernhard Nebel. Terminological reasoning is inherently Intractable. artificial Intelligence, forty 3:235-249, 1990.

10. Peter F. Patel-Schneider, Deborah L. McGuiness, Ronald J. Brachman, Lori Alperin Resnick, and Alexander Borgida. The traditional facts example gadget: Guiding ideas and Implementation rational. SIGART Bull., 2(3):108-113, 1991

11. Ronald J. Brachman. "lowering" traditional to practice: statistics example meets truth. In Proc. Of the 0.33 Int. Conf. at the ideas of information example and Reasoning (KR'ninety), Pp. 247- 258. Morgan Kaufmann, Los Altos, 1992.

12. Manfred Schmidt-Schau_ and Gert Smolka. Attributive concept descriptions with complements. Synthetic Intelligence, 48(1):1-26, 1991.

13. Francesco M. Donini, Maurizio Lenzerini, Daniele Nardi, and Werner Nutt. Thecomplexity of idea Languages. In James Allen, Richard Fikes, and Erik Sandewall,editors, Proc. Of the second Int. Conf. on the requirements of know-how instance and Reasoning (KR'ninety one), pp .151-162. Morgan Kaufmann, Los Altos, 1991.

14. Bernhard Hollunder, Werner Nutt, and Manfred SchmidtSchau_. Subsumption algorithms for idea Description languages. In Proc. Of the ninth Eur. Conf. On artificial Intelligence (ECAI'ninety), pp. 348-353, London (united kingdom), 1990 modified

15. Franz Baader and Bernhard Hollunder. A Terminological information instance tool with whole inference set of tips. In Proc. Of The Workshop on Processing Declarative data (PDK'ninety one), quantity 567 of Lecture Notes in synthetic Intelligence, pp. sixty seven-86. Springer-Verlag, 1991.

16. P. Bresciani, E. Franconi, and S. Tessaris. enforcing and locating out expressive description logics: initial file. In Proc. Of the 1995 Description particular judgment Workshop (DL'ninety five), pp.131-139, 1995.

17. Francesco M. Donini, Maurizio Lenzerini, Daniele Nardi, and Werner Nutt.Tractable idea languages. In Proc. Of the 12th Int. Joint Conf. On artificial Intelligence (IJCAI'91), pp. 458463, Sydney (Australia), 1991.

18. Francesco M. Donini, Bernhard Hollunder, Maurizio Lenzerini, Alberto Marchetti Spaccamela, Daniele Nardi, and Werner Nutt. The complexity of existential Quantification in concept languages. Synthetic Intelligence,pp.309-327, 1992. 
19. Francesco Donini and Fabio Massacci. EXPTIME Tableaux for ALC. Acta Informatica,124(1):87138, 2000.

20. Klaus Schild. A correspondence concept for Terminological logics: initial file. In Proc. Of The 12th Int. Joint Conf. On artificial Intelligence (IJCAI'ninety one), pp. 466-seventy one, 1991.

21. Ian Horrocks and Ulrike Sattler. An outline unique judgment With transitive and inverse roles and function hierarchies. J. Of not unusual experience and Computation, 9(3):pp.385-410, 1999.

22. Ian Horrocks, Ulrike Sattler, and Stephan Tobies. sensible reasoning for expressive description logics. In Harald Ganzinger, David McAllester, and Andrei Voronkov, editors, Proc. Of the 6th Int. Conf. On specific judgment For Programming and automated Reasoning (LPAR'ninety nine), variety 1705 in Lecture Notes in synthetic Intelligence, pages 161a hundred and eighty. Springer-Verlag, 1999.

23. Giuseppe De Giacomo and Maurizio Lenzerini. Boosting the correspondence amongst description Logics and propositional dynamic logics. In Proc. Of the twelfth countrywide. conference . On artificial Intelligence (AAAI'90 four), pp.205212. AAAI Press/The MIT Press, 1994

24. Giuseppe De Giacomo and Maurizio Lenzerini. idea language with variety policies and Xpoints, and its courting with calculus. In Proc. Of The11th Eur. Conf. On artificial Intelligence (ECAI'ninety 4), Pp. 411- 415, 1994.

25. Giuseppe De Giacomo. Decidability of sophistication-based completely statistics instance Formalisms. PhD thesis, Dipartimento di Informatics e Sistemistica, Universit_a Di Roma 1. A. Sapienza", 1995

26. Giuseppe De Giacomo and Maurizio Lenzerini. TBox And ABox reasoning in expressive description logics. In Luigia C. Aiello, John Doyle, and Stuart C. Shapiro, Editors, Proc. Of the fifth Int. Conf. at the necessities of facts example and Reasoning (KR'ninety six), Pp. 316-327. Morgan Kaufmann, Los Altos, 1996

27. Ian Horrocks. using an expressive description proper judgment: fact or Fiction? In Proc. Of the 6th Int. Conf. On requirements of data example and Reasoning (KR'98), pp. 636-647, 1998

28. Volker Haarslev and Ralf $\mathrm{M} \square$ oller. RACE device Description. In Proc. Of the 1999 Description suitable judgment Workshop (DL'ninety nine), pages a hundred thirty132. CEUR virtual Workshop court instances, http://ceur-Ws.Org/Vol-22/, 1999.

29. Peter F. Patel-Schneider. DLP. In Proc. Of the 1999 Description correct judgment Workshop (DL'99), pages 9thirteen CEUR electronic Workshop courtroom instances, Http://ceurws. Org/Vol-22/, 1999.

30. Alexander Borgida. on the relative expressiveness of Description logics and predicate logics. Synthetic Intelligence, 80 (12):353367, 1996.

31. Leszek Pacholski, Wieslaw Szwast, and Lidia Tendera. Complexity of -variable suitable judgment with counting. In intending of the 12th IEEE Symp. On common revel in in pc generation (LICS'ninety seven), pages 318327. IEEE laptop Society Press, 1997

32. Erich Gr $\square$ adel, Phokion G. Kolaitis, and Moshe Y. Vardi. At the choice trouble for 2-variable _rst-order common feel. Bulletin of Symbolic appropriate judgment, three(1):fifty threesixty nine, 1997.

33. Erich Gr., Guarded fragments of first-order actual judgment: A mind-set for latest description logics? In Proc. Of the 1998 Description not unusual experience Workshop (DL'98). CEUR virtual Workshop court docket times, http://ceurWs.Org/Vol-11/, 1998.

34. Erich Gr., on the restraining power of guards. J. Of Symbolic commonplace experience, sixty four:pp. 1719-1742, 1999.

35. Ullrich Hustadt, Renate A. Schmidt, and Lilia Georgieva. A survey of decidable first-order fragments and outline logics. mag of Relational strategies in laptop era, 1:251276, 2004.

36. Martin Buchheit, Francesco M. Donini, Werner Nutt, And Andrea Schaerf. form for terminological structures: Terminology $=$ schema + views. synthetic Intelligence, ninety nine(2):pp.209-260, 1998.

37. Diego Calvanese, Giuseppe De Giacomo, and Maurizio Lenzerini. at the decidability of query containment beneath constraints. In Proc. Of the 17th ACM SIGACT SIGMOD SIGART Symp. On thoughts of Database structures (PODS'ninety eight), pp.149-158, 1998.

38. Diego Calvanese, Giuseppe De Giacomo, Maurizio Lenzerini, Daniele Nardi, and Riccardo Rosati. Description not unusual revel in framework for information Integration. In proceeding of the sixth Int. Conf. On necessities of information instance and Reasoning (KR'ninety eight), pages 2thirteen, 1998.

39. Bernardo Cuenca Grau, Ian Horrocks, Yevgeny Kazakov, and Ulrike Sattler. A logical framework for Modularity of ontologies. In Manuela M. Veloso, Editor, Proc. Of the 20th Int. Joint Conf. On artificial Intelligence (IJCAI 2007), pp. 298-303, Hyderabad, India, 2007.

40. Evren Sirin and Bijan Parsia. Pellet: An OWL DL Reasoner. In Proc. Of the 2004 Description common revel in Workshop (DL 2004), pp. 212- 213, 2004

41. Ullrich Hustadt and Renate A. Schmidt. on the relation Of desire and tableaux proof systems for description Logics. In Proc. Of the sixteenth Int. Joint Conf. On synthetic Intelligence (IJCAI'ninety nine), pp. a hundred and ten-117, 1999.

42. Ullrich Hustadt and Renate A. Schmidt. problems of Decidability for description logics in the framework of decision. In R. Caferra and G. Salzer, editors, computerized duction in Classical and Non-Classical Logics, amount 1761 of Lecture Notes in synthetic Intelligence, pages 191205. Springer-Verlag, 2000.

43. Ullrich Hustadt, Boris Motik, and Ulrike Sattler. decreasing SHIQ-description proper judgment to disjunctive Datalog packages. In Didier Dubois, Christopher A. Welty,and MaryAnne Williams, editors, Proc. Of the ninth Int. Conf. On mind of expertise example and Reasoning (KR 2004), pp. Fifty 162. Morgan Kaufmann, Los Altos, 2004.

44. Yevgeny Kazakov and Boris Motik. A preference- based totally desire method for SHOIQ. In Ulrich Furbach and Natarajan Shankar, editors, Proc. Of the Int. Joint Conf. On automatic Reasoning (IJCAR 2006), amount 4130 of Lecture Notes In pc technological facts, pages 662677. SpringerVerlag, 2006.

45. Carsten Lutz and Ulrike Sattler. Mary likes all cats. In Proc. Of the 2000 Description remarkable judgment Workshop (DL 2000), pp. 213-226.

46. Carsten Lutz. c language-based simply temporal reasoning with stylish TBoxes. In Proc.Of the seventeenth Int. Joint Conf. On artificial Intelligence (IJCAI 2001), pp. 89-ninety four, 2001

47. Franz Baader and Stephan Tobies. The inverse technique Implements the automata approach for modal Satisfiability. In Proc. Of the Int. Joint Conf. On automatic Reasoning (IJCAR 2001), volume 2083 of Lecture Notes in artificial Intelligence, pp. ninety -106. Springer-Verlag, 2001.

48. Franz Baader, Jan Hladik, Carsten Lutz, and Frank Wolter. From tableaux to automata for description Logics. critical Informatica, 57(24) pp.247-279, 2003

49. Ian Horrocks, Oliver Kutz, and Ulrike Sattler. The even more no longer possible to stand as much as SROIQ. In Patrick Doherty, John Mylopoulos, and Christopher A. Welty, editors, Proc. Of the 10th Int. Conf. On mind of know-how instance and Reasoning (KR 2006), pp. 57-sixty seven, Lake District, uk, 2006. AAAI Press/The MIT Press. 
50. Franz Baader, Sebastian Brandt, and Carsten Lutz. Pushing the EL envelope. In Leslie \% Kaelbling and Alessandro Sa_otti, editors, Proc. Of the nineteenth Int. Joint Conf. On Arti_cial Intelligence (IJCAI 2005), Pp. 364-369, Edinburgh (united kingdom), 2005. Morgan Kaufmann, Los Altos.

51. Franz Baader, Sebastian Brandt, and Carsten Lutz. Pushing the EL envelope similarly. In Kendall Clark and Peter F. Patel-Schneider, editors, In court docket times of the fifth global Workshop on OWL: evaluations and recommendations (OWLED'08), Karlsruhe, Germany, 2008.

52. Michael J. Fischer and Richard E. Ladner. Propositional dynamic nicely judgment of regular applications. J. Of laptop and machine Sciences, 18:pp.194-211, 1979

53. Diego Calvanese, Giuseppe De Giacomo, Domenico Lembo, Maurizio Lenzerini, and Riccardo Rosati. Tractable reasoning and green query answering in Description logics: The DL-Lite family. J. Of automated Reasoning, 39(three):pp.385429,2007.

54. Birte Glimm, Ian Horrocks, Carsten Lutz, and Ulrike Sattler. Conjunctive question answering for the Description not unusual enjoy SHIQ. In Manuela M. Veloso, editor, Proc. Of 20th Int. Joint Conf. On artificial Intelligence (IJCAI 2007), pages 399404, Hyderabad, India, 2007.

55. Carsten Lutz. The complexity of conjunctive query Answering in expressive description logics. In Alessandro Armando, Peter Baumgartner, and Gilles Dowek, editors, Proc. Of the Int. Joint Conf. On automated Reasoning (IJCAR 2008), Lecture Notes in artificial Intelligence, pages 179193. Springer-Verlag, 2008.

56. Magdalena Ortiz, Diego Calvanese, and Thomas Eiter. facts complexity of question answering in expressive Description logics thru tableaux. J. Of computerized Reasoning, forty one(1):pp.61-98, 2008

57. Stefan Schlobach and Ronald Cornet. Non-famous Reasoning offerings for the debugging of description right judgment terminologies. In Georg Gottlob and Toby Walsh, Editors, Proc. Of the 18th Int. Joint Conf. On artificial Intelligence (IJCAI 2003),pages 355362, Acapulco, Mexico, 2003.

58. Bijan Parsia, Evren Sirin, and Aditya Kalyanpur. Debugging OWL ontologies. In Allan Ellis and Tatsuya Hagino, editors, Proc. Of the 14th international convention on international huge internet (WWW'05), pp. 633-640. ACM, 2005.

59. Thomas Meyer, Kevin Lee, Richard earnings place, and Je_Z. Pan. finding maximally satisfiable terminologies for The outline particular judgment ALC. In Proc. Of the 21st Nat. Conf. On Arti_cial Intelligence (AAAI 2006). AAAI Press/The MIT Press, 2006.

60. Franz Baader, Rafael Pe naloza, and Boontawee Suntisrivaraporn. Pinpointing inside the description unique judgment EL+. In Proc. Of the thirtieth German Annual Conf. On synthetic Intelligence (KI'07), amount 4667 of Lecture Notes in synthetic Intelligence, pp. Fifty -67, Germany, 2007.

61. Boontawee Suntisrivaraporn. Module extraction and Incremental beauty: a practical method for EL+ ontologies. In Sean Bechhofer, Manfred Hauswirth,Joerg Ho_mann, and Manolis Koubarakis, Editors, court docket times of the fifth ecu Semantic net convention (ESWC'08), quantity 5021 of Lecture Notes in pc generation, pp. 230-244, Springer-Verlag, 2008.

62. Franz Baader, G.Brewka and Oliver F. Gill, which incorporates Threshold mind to the outline properly judgment EL , Dresden college, 2008

63. Mike Bergman, essential significance of retaining an ABox and TBox split, AAAI, 2009

64. Jean-Baptsitc Lamy, OWL ready : Ontology - oriented Programming in Python with computerized elegance And excessive degree constructs for natural ontologies, 2007

65. Wordelt Sebastian and Moller R, inside the direction of a topic Modularization of semi-expressive DLs, IOS Press 2012

66. H. Peter Alesso and Craig F. Smith : questioning at the internet - Wiley India , 2009
67. Berners-Lee $\mathrm{T}$, Hendler $\mathrm{J}$ and Ora L: The semantic net, medical American, also can 2001 pp. 35-forty three

68. Modal common feel,Stanford Encyclopedia of Philosophy.Html, 2014 